\title{
Treating Severe Plasmodium falciparum Malaria in the United States: Historical Trends and Current Challenges
}

\author{
Albert Anastasio*, Kevin Farley \\ Emory University School of Medicine, Atlanta, Georgia, USA
}

Article Info

Article Notes

Received: September 9, 2018

Accepted: September 26, 2018

\section{*Correspondence:}

Dr. Albert Anastasio, 1648 Pierce Dr NE, Atlanta, GA 30307, USA; Email: albert.anastasio@emory.edu

${ }^{0} 2018$ Anastasio A. This article is distributed under the terms of the Creative Commons Attribution 4.0 International License.

\section{Introduction}

Malaria, a parasitic disease transmitted by the Anopheles mosquito, remains a cause of great morbidity and mortality worldwide. Numbers of reported cases from the most recent report on malaria surveillance in the United States (US) shows a continual drop in the burden of malarial disease nationally $(1,727$ and 1,725 in 2013 and 2014 to 1,517 in 2015) a 1-4. Plasmodium falciparum constituted $67.4 \%$ of infections and a reported $17.1 \%$ of malaria infections were classified as "severe illnesses"3. Despite reports of dropping incidence over the last few years, the number of malaria deaths in the United States in 2015 was actually higher (11 deaths) than the average over the years 2000-2014 (6.1 deaths per year) ${ }^{3}$. This brief report discusses the current trends in the treatment of severe malaria in the US. Articles were found in pub-med indexed journals by a team of investigators.

\section{Early Stage of the Disease}

While this article will focus on the treatment of severe malaria (described below) in the US, it is critical to understand the early stages of the disease in an effort to prevent progression to the severe form of malaria. The early symptoms of malaria are very nonspecific, and so returning travelers often hesitate to seek medical counsel. Initially, headache, malaise, muscle aches, and abdominal discomfort may be experienced, followed by a characteristic irregular fever ${ }^{4}$. However, there is no specific set of symptoms for malaria, and the disease can present variably ${ }^{4,5}$. Mild anemia, hepatosplenomegaly, and mild jaundice may also accompany these early symptoms ${ }^{4}$. When uncomplicated malaria is diagnosed and treated quickly and appropriately, mortality rates are extremelylow ${ }^{4}$. In addition to serum antibody testing, diagnosis is obtained through examination of thick and thin blood films by an expert trained to determine speciation of the parasite and current parasite load ${ }^{5}$. Early hospitalization is recommended. Initial treatment differs by country. In the United Kingdom (UK), the initial treatment for uncomplicated malaria is Artemether-lumefantrine with dihydroartemisinin-piperaquine as an alternative therapy ${ }^{\mathrm{b}}{ }^{5}$. In the US, the first line treatment is chloroquine phosphate or hydroxychloroquine according to the 2013 Centers for Disease Control (CDC) Guidelines for Treatment

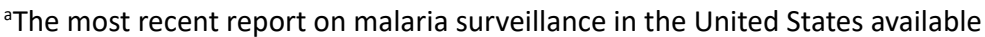
on PubMed is the 2015 report. Final publication of these reports typically lags three years behind collection of data.

${ }^{b}$ Both of these medications are artemesinin derived therapies, in stark contrast to first line medication in the US 
of Malaria ${ }^{6}$. This difference in initial treatment is a major point of distinction between treatment of malaria in the UK and the US, as we will explore when we discuss the treatment of severe malaria.

\section{Progression to "Severe Malaria"}

If initiation of treatment is delayed or not completed soon enough and parasite burden progresses, uncomplicated malaria can develop into severe malaria ${ }^{4,7,8}$. The clinical syndrome of severe malaria constitutes a series of clinical signs and laboratory values indicating worsening illness and end-organ dysfunction ${ }^{4}$. Clinical symptoms consist of prostration, confusion or agitation, coma, respiratory distress, convulsions, shock (prolonged capillary refill time $>2 \mathrm{~s}$ ), pulmonary edema, abnormal bleeding, jaundice, anuria, and repeated vomiting ${ }^{4}$. Laboratory values seen in severe malaria include hemoglobin $<8 \mathrm{~g} / \mathrm{dl}$, hemoglobinuria, hypoglycemia $<40 \mathrm{mg} / \mathrm{dl}$, acidosis (base deficit $>8 \mathrm{meq} / \mathrm{L}$ or plasma bicarbonate $<15 \mathrm{mmol} / \mathrm{L}$ ), acute kidney injury (creatinine $>3 \mathrm{mg} / \mathrm{dL}$ or urea $>20$ $\mathrm{mmol} / \mathrm{L})$, and asexual parasitemia $>10 \%$ of infected red blood cells ${ }^{4}$. Once progression to severe malaria has occurred, intravenous (IV) antiparasitic agents must be administered. Consensus on choice of treatment in the UK is Intravenous artesunate $(2.4 \mathrm{mg} / \mathrm{kg}$ per dose hours $0,12$, and 24 ; then every $24 \mathrm{~h})^{4,5,9}$. Artesunate is also the treatment of choice in Germany ${ }^{10}$.

\section{History of Treatment of Severe Malaria in the US}

A review article published in 1972 noted that roughly 3,000 cases of malaria were reported annually in the US. That article describes the same clinical course for severe malaria seen today, with renal, cerebral, pulmonary, and hematologic complications. For uncomplicated cases, quinine was the treatment of choice ${ }^{8}$. For severe malaria, $650 \mathrm{mg}$ of quinine dihydrochloride diluted in saline solution and administered intravenously was considered the first line treatment ${ }^{8}$. In 1991, the CDC issued a guideline for the treatment of severe malaria recommending the treatment of Plasmodium falciparum malaria with IV quinidine gluconate ${ }^{11}$. A stereoisomer of quinine, IV quinidine gluconate at a dose of $6.25 \mathrm{mg}$ base $/ \mathrm{kg} \quad(=10$ mg salt $/ \mathrm{kg}$ ) loading dose IV over $1-2 \mathrm{hrs}$, then $0.0125 \mathrm{mg}$ base $/ \mathrm{kg} / \mathrm{min}$ (=0.02 $\mathrm{mg}$ salt $/ \mathrm{kg} / \mathrm{min}$ ) continuous infusion for at least 24 hours remains the first line treatment for severe Plasmodium falciparum malaria in the US6. Administration of this drug should be accompanied by cardiac and blood pressure monitoring for widening of the QRS complex and/or lengthening of the QTc interval and hypotension respectively ${ }^{6}$. For severe malaria, IV quinidine gluconate should be accompanied with one of the following adjunct treatments: doxycycline: $100 \mathrm{mg}$ po bid x 7 days, tetracycline: $250 \mathrm{mg}$ po qid $x 7$ days, or clindamycin: $20 \mathrm{mg}$ base $/ \mathrm{kg} /$ day po divided tid $x 7$ days ${ }^{6}$.

\section{Current Treatment Protocol in the US}

No article which specifically outlines the treatment protocol of severe malaria in the United States has been published. The most recent review article published on the treatment of malaria in general in the United States dates from 2007. The authors write: "The artemesinin derivatives clear parasites very rapidly, are now a key component of malaria treatment worldwide, and have been shown to reduce mortality in severe malaria compared with parenteral quinine. These drugs are not yet available in the United States, but the CDC hopes to make intravenous artesunate available under an Investigational New Drug protocol in 2007"7.

In 2018, over 10 years after this review article was published, quinidine gluconate remains the first line treatment for severe malaria ${ }^{6}$, despite growing bodies of evidence that assert the superiority of artemesinin derived antiparasitics ${ }^{4,12-15}$. The CDC did hold true to their promise, however, and artesunate is currently available as an investigational drug from designated CDC outposts throughout the country. Many reports of the favorable use of artesunate in the US exist, but the drug is still unavailable on hospital formularies ${ }^{15-17}$. Obtaining a waiver from the CDC and arranging for transportation of the medication would delay initiation of artemesinin therapy leading to potential progression of the disease, perhaps explaining rising malaria death rates in 2015 , especially as resistance to quinine escalates among returning travelers ${ }^{18-22}$.

Why has the US lagged behind other countries in widespread adoption of artemisinin based for treatment of severe malaria? Several reports of delayed hemolysis after use of artemesinins exist. This phenomenon presents 1-3 weeks after artemesinin-containing antiparasitics are begun and manifests as a decline in hemoglobin potentially severe enough to warrant transfusion ${ }^{23-27}$. A review article published in 2015 reports rates of $7-21 \%$ for development of post artemesinin delayed hemolysis (PADH) ${ }^{28}$. Recent reports of PADH show full recovery after diagnosis. In a Canadian case report, 2 returning travelers developed PADH. Both were treated with transfusion of packed red blood cells (pRBCs) and their anemia resolved ${ }^{23}$. Paczkowski et al. report 2 cases in the US between 2012 and 2014 in addition to the 18 cases identified internationally by the CDC. Of the patients who could be reached for follow up of these 20 cases, all experienced full recovery although several received transfusion of $\mathrm{pRBCs}^{26}$. the authors conclude that artesunate remains a safe and effective option for severe malaria ${ }^{26}$. Additionally, researchers have identified a test which can predict potential for development of PADH. The histidine-rich protein 2 (HRP2) predicted subsequent PADH with $89 \%$ sensitivity and $73 \%$ specificity ${ }^{29}$. This lab test could identify patients at risk for PADH in addition to recommended screening for at least 4 weeks after 
discharge to detect symptomatic hemolysis ${ }^{28}$. Artesunate is a safe and effective therapy for severe malaria, but, as with any medications appropriate precautions should be maintained.

\section{Supplemental Therapy to Prevent Organ Failure}

While antiparasitic agents formulate the backbone of treatment for severe malaria, controlling deterioration of organ function via other supportive therapies is critical to reduce mortality in life-threatening illness. First off, careful investigation should be undertaken to ensure end-organ dysfunction is the result of a malaria infection and not a concomitant infection by another organism. Endovascular dysfunction leading to microvascular occlusion is thought to be the main cause of end-organ dysfunction in severe malaria, although precise pathogenesis and additional mechanisms are a topic of much research ${ }^{30-35}$. Cerebral malaria, severe anemia, hypoglycemia, pulmonary edema, and kidney dysfunction constitute the primary manifestations of end-organ damage in severe malaria ${ }^{4}$. An in-depth management guide for each of these critical pieces in malaria management is outside the scope of this brief article, but several critical interventions should be pursued should such conditions present in the patient with severe malaria.

Cerebral malaria presents as neurologic dysfunction and eventual loss of consciousness. While no specific consensus protocol exists on the treatment of this condition, prompt anti-malarial therapy as well as attention to management of hypoglycemia, acidosis and hypovolemia is beneficial ${ }^{36}$. Lumbar puncture should be performed to rule out bacterial meningitis and MRI should be performed to rule out cerebral edema or bleed ${ }^{7}$. A number of experimental therapies have been proposed with mixed results ${ }^{36-39}$. Severe anemia from repeated bouts of malaria in pregnant women and children is a major issue in endemic countries. Due to transfusion carrying a risk for HIV transmission in countries where malaria is endemic, transfusion for anemia related to malaria is a topic of current debate ${ }^{40,41}$. While the CDC does not recommend exchange transfusion for treatment of malaria, in areas where risk of HIV transmission through blood transfusion is lower, transfusion has been used successfully in some case reports ${ }^{42,43}$.

Hypoglycemia can be masked by the symptoms of cerebral malaria, and so regular monitoring of blood glucose is indicated ${ }^{7}$. This entity can be induced by hyperinsulinemia following administration of quinidine ${ }^{7}$. Appropriate fluid replacement and renal replacement therapy is the mainstay of treatment for acute kidney injury from severe malaria ${ }^{44,45}$. Special care must be taken to avoid nephrotoxic drugs and vasodilators appear to have no survival benefit ${ }^{44}$. Pulmonary edema is either the result of impaired kidney function or development of acute respiratory distress syndrome in severe malaria ${ }^{7}$. Keeping the patient euvolemic is imperative and if needed, the use of endotracheal intubation and positive end-expiratory pressure ventilation should be considered ${ }^{7,46}$.

\section{Conclusions}

Severe malaria is a rare but treatable condition that affects a slowly declining number of returning travelers in the US. The majority of the world uses artemesinin derived antiparasitics as the first line treatment for severe malaria, but the US uses IV parenteral quinidine accompanied by an antibiotic. Artesunate is available from the CDC for use as an experimental agent. Post artemesinin delayed hemolysis is a recognized clinical entity, and patients should be followed up appropriately for development of this condition, but this phenomenon should not preclude the use of artemesinin derived antiparasitics. Supportive treatment to prevent end-organ damage should be initiated when necessary in cases of severe malaria.

\section{References}

1. Cullen KA, Mace KE, Arguin PM. Malaria Surveillance - United States, 2013. MMWR Surveill Summ. 2016; 65(2): 1-22.

2. Mace KE, Arguin PM. Malaria Surveillance - United States, 2014. MMWR Surveill Summ. 2017; 66(12): 1-24.

3. Mace KE, Arguin PM, Tan KR. Malaria Surveillance - United States, 2015. MMWR Surveill Summ. 2018; 67(7): 1-28.

4. White NJ. Malaria. Lancet. 2014; 383(9918): 723-35.

5. Lalloo DG, Shingadia D, Bell DJ, et al. UK malaria treatment guidelines 2016. J Infect. 2016; 72(6): 635-649.

6. Centers for Disease Control: Guidelines for Treatment of Malaria in the United States. 1-3.

7. Griffith KS, Lewis LS, Mali S, et al. Treatment of malaria in the United States: a systematic review. JAMA. 2007; 297(20): 2264-77.

8. Heineman HS. The clinical syndrome of malaria in the United States. A current review of diagnosis and treatment for American physicians. Arch Intern Med. 1972; 129(4): 607-16.

9. Askling HH. Management of imported malaria in Europe. Malar J. 2012; 11: 328.

10. Vinnemeier CD, Rolling T. [Prophylaxis and Therapy of Malaria: Current Recommendations]. Dtsch Med Wochenschr. 2018; 143(7): $472-475$.

11. Centers for Disease C. Treatment of severe Plasmodium falciparum malaria with quinidine gluconate: discontinuation of parenteral quinine from CDC drug service. MMWR Morb Mortal Wkly Rep. 1991; $40(14): 240$.

12. Hess KM, Goad JA, Arguin PM. Intravenous artesunate for the treatment of severe malaria. Ann Pharmacother. 2010; 44(7-8): 12508.

13. Jones KL, Donegan S, Lalloo DG. Artesunate versus quinine for treating severe malaria. Cochrane Database Syst Rev. 2007; (4): CD005967.

14. Sinclair D, Donegan S, Isba R, et al. Artesunate versus quinine for treating severe malaria. Cochrane Database Syst Rev. 2012; (6): CD005967.

15. Twomey PS, Smith BL, McDermott C, et al. Intravenous Artesunate for the Treatment of Severe and Complicated Malaria in the United 
States: Clinical Use Under an Investigational New Drug Protocol. Ann Intern Med. 2015; 163(7): 498-506.

16. Callender DM, Hsue G. Artesunate: investigational drug for the treatment of severe falciparum malaria in Hawai'i. Hawaii Med J. 2011; 70(4): 77-9.

17. Shah PJ, Koshy J, Everett N, et al. Severe Plasmodium falciparum Malaria Treated With Investigational Artesunate in the United States. J Pharm Pract. 2018; 897190018782367.

18. Bertaux L, Kraemer P, Taudon N, et al. Quinine-resistant malaria in traveler returning from French Guiana, 2010. Emerg Infect Dis. 2011; 17(5): 943-5.

19. Jelinek T, Schelbert P, Löscher T, et al. Quinine resistant falciparum malaria acquired in east Africa. Trop Med Parasitol. 1995; 46(1): 3840.

20. Pradines B, Pistone T, Ezzedine K, et al. Quinine-resistant malaria in traveler returning from Senegal, 2007. Emerg Infect Dis. 2010; 16(3): 546-8.

21. Rodríguez-Valero N, Camprubí D, García-Guijarro E, et al. Suspected quinine resistant $P$. falciparum severe malaria possibly acquired in Ivory Coast. Parasitol Int. 2018; 67(6): 684-687.

22. Uchiyama H, Okamoto A, Sato K, et al. Quinine-resistant severe falciparum malaria effectively treated with atovaquone and proguanil hydrochloride combination therapy. Intern Med. 2004; 43(7): 624-7.

23. Charles M, Patterson JM, Asadi L, et al. Delayed Hemolysis After Parenteral Artesunate Therapy for Severe Malaria in Two Returning Canadian Travelers. Am J Trop Med Hyg. 2015; 93(4): 819-21.

24. Clark RL. Hypothesized cause of delayed hemolysis associated with intravenous artesunate. Med Hypotheses. 2014; 82(2): 167-70.

25. Lahoud JS, Lahoud OB, Lin YS, et al. Artesunate-related fever and delayed hemolysis in a returning traveler. IDCases. 2015; 2(2): 63-5.

26. Paczkowski MM, Landman KL, Arguin PM. Update on cases of delayed hemolysis after parenteral artesunate therapy for malaria - United States, 2008 and 2013. MMWR Morb Mortal Wkly Rep. 2014; 63(34): 753-5.

27. Rolling $\mathrm{T}$, Agbenyega $\mathrm{T}$, Issifou $\mathrm{S}$, et al. Delayed hemolysis after treatment with parenteral artesunate in African children with severe malaria--a double-center prospective study. J Infect Dis. 2014 209(12): 1921-8.

28. Rolling T, Agbenyega T, Krishna $\mathrm{S}$, et al. Delayed haemolysis after artesunate treatment of severe malaria - review of the literature and perspective. Travel Med Infect Dis. 2015; 13(2): 143-9.

29. Ndour PA, Larréché $S$, Mouri $O$, et al. Measuring the Plasmodium falciparum HRP2 protein in blood from artesunate-treated malaria patients predicts post-artesunate delayed hemolysis. Sci Transl Med. 2017; 9(397).

30. Dondorp AM, Nyanoti M, Kager PA, et al. The role of reduced red cell deformability in the pathogenesis of severe falciparum malaria and its restoration by blood transfusion. Trans R Soc Trop Med Hyg. 2002; 96(3): 282-6.

31. Ferreira A, Balla J, Jeney V, et al. A central role for free heme in the pathogenesis of severe malaria: the missing link. J Mol Med (Berl). 2008; 86(10): 1097-111.

32. Liles WC, Kain KC. Endothelial activation and dysfunction in the pathogenesis of microvascular obstruction in severe malaria--a viable target for therapeutic adjunctive intervention. J Infect Dis. 2014; 210(1): 163-4.

33. Mackintosh CL, Beeson JG, Marsh K. Clinical features and pathogenesis of severe malaria. Trends Parasitol. 2004; 20(12): 597-603.

34. Wassmer SC, Grau GE. Severe malaria: what's new on the pathogenesis front. Int J Parasitol. 2017; 47(2-3): 145-152.

35. Wassmer SC, Taylor TE, Rathod PK, et al. Investigating the Pathogenesis of Severe Malaria: A Multidisciplinary and Cross-Geographical Approach. Am J Trop Med Hyg. 2015; 93(3 Suppl): 42-56.

36. Golenser J, McQuillan J, Hee L, et al. Conventional and experimental treatment of cerebral malaria. Int J Parasitol. 2006; 36(5): 583-93.

37. Burton A. New hope for cerebral malaria treatment. Lancet Neurol. 2005; 4(10): 600.

38. El-Assaad F, Combes V, Grau GE, et al. Potential efficacy of citicoline as adjunct therapy in treatment of cerebral malaria. Antimicrob Agents Chemother. 2014; 58(1): 602-5.

39. Wang W, Qian H, Cao J. Stem cell therapy: a novel treatment option for cerebral malaria? Stem Cell Res Ther. 2015; 6: 141.

40. Ekvall H. Malaria and anemia. Curr Opin Hematol. 2003; 10(2): 10814.

41. Nwaneri MO. Transfusion of blood components may improve survival in severe malaria anemia in children. Am J Trop Med Hyg. 2008; 78(5): 693; author reply 693-4.

42. Ou TY, Chuang CY, Chen CD, et al. Therapeutic plasma exchange in the treatment of complicated Plasmodium falciparum malaria: A case report. J Clin Apher. 2017.

43. Antinori S, Corona A, Castelli A, et al., Severe Plasmodium falciparum malaria in the intensive care unit: A 6-year experience in Milano, Italy. Travel Med Infect Dis. 2017; 17: 43-49.

44. Das BS. Renal failure in malaria. J Vector Borne Dis. 2008; 45(2): 8397.

45. Meremo AJ, Kilonzo SB, Munisi D, et al. Acute renal failure in a Caucasian traveler with severe malaria: a case report. Clin Case Rep. 2014; 2(3): 82-5.

46. von Sonnenburg F, Löscher T, Nothdurft HD, et al. [Complicated malaria tropica: specific and supportive therapy in the imported diseases]. Dtsch Med Wochenschr. 1986; 111(24): 934-8. 